\title{
PHOTOPLASTIC SU-8 PROBES FOR NEAR-FIELD OPTICAL APPLICATIONS
}

\author{
Jürgen Brugger ${ }^{1}$, Beomjoon Kim ${ }^{1}$ and Niek van Hulst ${ }^{2}$ \\ Strategic Research Orientation NanoLink ${ }^{1} \&$ Applied Optics Group ${ }^{2}$ \\ MESA ${ }^{+}$Research Institute, University of Twente \\ P.O. Box 217, 7500 AE Enschede, The Netherlands, e-mail: j.p.brugger@el.utwente.nl
}

\begin{abstract}
Scanning Near-field Optical Microscopy (SNOM) overcomes the diffraction limit of resolution and has hence become a widely used technique for the optical investigation of materials in the sub-wavelength range. However, the technique's further advancement, especially in the field of biology and surface chemistry is hampered mainly due to the unavailability of inexpensive high-quality probes, which is in contrast to the success story of the atomic force microscope (AFM) during the past decade. Low-cost manufacturing of highquality SNOM probes is still unsolved albeit several methods for integrated manufacturing have been proposed in the past ${ }^{1,}$.
\end{abstract}

We propose here a new attempt to solve the manufacturing problem by a novel waferscale microfabrication process for sharp pyramidal and bright photo-plastic probes. The fabrication steps are adapted from a recently proposed technique to make low-cost AFM probes $^{3}$. The probes are fabricated of a transparent photo-plastic material (SU-8) which allows simple batch fabrication based on spin coating and subsequent near-ultraviolet exposure and development steps. SU-8, an epoxy-based, negative tone, near-ultraviolet (UV) photoresist is used increasingly in the growing field of micro-electro-mechanicalsystems (MEMS) as photoresist as well as structural material ${ }^{4}$. It consists of the epoxy-based EPON SU-8 resin photosensitized with a triaryl sulfonium salt. For MEMS applications, the main interest feature of the resist includes the possibility to define high aspect ratio structures at low-cost, without the need for an expensive x-ray source (as e.g. required in conventional LIGA). The main interest for MOEMS applications is that SU-8 is transparent. These combined advantages are used here to define a sharp, transparent and high aspect ratio probe dedicated for near-field optical applications.

The process starts with the fabrication of molds in a (100) oriented silicon wafer by using the anisotropic etchant potassium-hydroxide. This self-limiting process results in inverted sharp pyramids limited by four $\langle 111\rangle$ silicon crystal planes. The cone angle is in the order of $\sim 54^{\circ}$. After the mold is covered with a thin $(<100 \mathrm{~nm})$ evaporated metal film, the photo-plastic material SU-8 is conformally spin-coated and structured by simple lithography. The cup-like probes are designed to incorporate standard optical fiber, which are aligned and bonded to the probes using optical adhesive (NOA73). The glue is cured by ultraviolet light and provides good optical index matching. The probes are then removed from the mold together with the metal layer. We have evidence that in our case the aluminum layer is considerably smoother compared to the 'grainy' layers typically evaporated on freestanding probes. This has been confirmed by atomic force microscope analysis. Therefore we expect to be able to reduce their thickness, which is advantageous for the probe geometry. A focussed ion beam (FIB) milling step finally forms the nanoscale aperture. Figure 1 shows the fabrication process for the proposed SNOM probe using micromachining technology. The schematic figure and SEM image of the microassembled probe is shown in Figure 2. The advantage of our method is that existing fiber-based SNOM setups can be used without modification. We will present details of the probe geometry, its fabrication process and first optical experiments. 


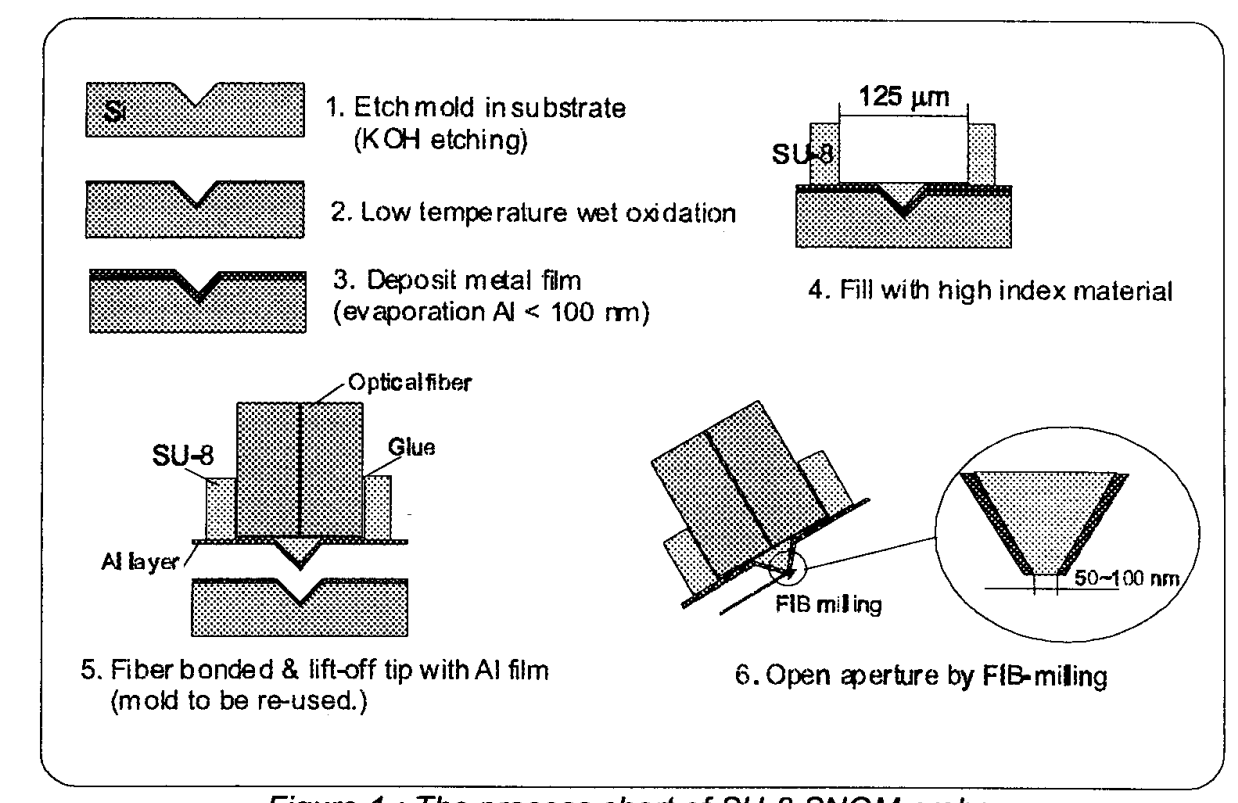

Figure 1 : The process chart of SU-8 SNOM probe.

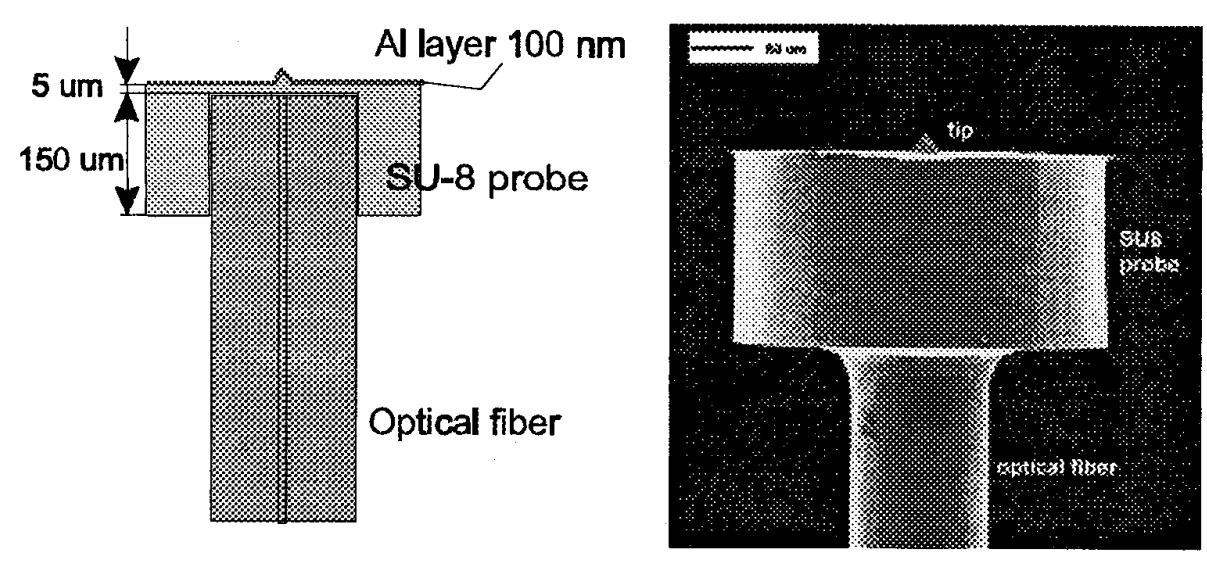

Figure 2 : Schematic (left) and SEM micrograph (right) of photoplastic SNOM probe.

\footnotetext{
' M. Abraham, W. Ehrfeld, M. Lacher, K. Mayr, W. Noell, P. Guthner, J. Barenz, " Micromachined aperture probe tip for multifunctional scanning probe microscopy", Ultramicoscopy, 71 (1998) 93-98. ${ }^{2}$ G. Schuermann, P.F. Indermuehle, U. Staufer, N.F.de Rooij, Micromachined SPM probes with sub$100 \mathrm{~nm}$ features at tips apex, Surface and Interface Analysis, 27, 299 (1999)

${ }^{3}$ G. Genolet, J. Brugger, M. Despont, U. Drechsler, P. Vettiger, N. F. de Rooij, and D. Anselmetti, " Soft, Entirely Photoplastic Probes for Scanning Force Microscopy ", Rev.Sci.Instr., 70(5) (1999) 2398 ${ }^{4}$ H. Lorenz, M. Despont, N. Fahrni, J. Brugger, P. Renaud, and P. Vettiger, Sens. Actuators A 64, 33 (1998)
} 